\title{
Role of Artificial Neural Networks in Pharmaceutical Sciences
}

\author{
Talasila Bhanu Teja ${ }^{1}$, Mahendran Sekar ${ }^{2, *}$, Talasila Pallavi ${ }^{3}$, Sivamma Mettu ${ }^{4}$, TE Gopalakrishna Murthy ${ }^{5, *}$, Nur Najihah Izzati Mat Rani ${ }^{6}$, \\ Pallaval Veera Bramhachari' ${ }^{7}$, Srinivasa Reddy Bonam ${ }^{8}$ \\ 'School of Computer Science and Engineering, Vellore Institute of Technology (VIT), Vellore, Tamil Nadu, INDIA.

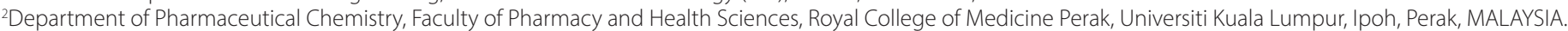 \\ ${ }^{3}$ Department of Computer Science and Engineering, KL University (Deemed to be University), Guntur, Andhra Pradesh, INDIA \\ ${ }^{4}$ Independent Researcher, Île-de-France, Paris, FRANCE. \\ ${ }^{5}$ Department of Pharmaceutics, Bapatla College of Pharmacy, Bapatla, Guntur, Andhra Pradesh, INDIA. \\ ${ }^{6}$ Faculty of Pharmacy and Health Sciences, Royal College of Medicine Perak, Universiti Kuala Lumpur, Ipoh, Perak, MALAYSIA \\ ${ }^{7}$ Department of Biotechnology, Krishna University, Machilipatnam, Andhra Pradesh, INDIA. \\ ${ }^{8}$ Institut National de la Santé et de la Recherche Médicale; Centre de Recherche des Cordeliers, Equipe-Immunopathologie et Immunointervention Thérapeutique, \\ Sorbonne Université, Université de Paris, Paris, FRANCE.
}

\begin{abstract}
Artificial Neural Networks (ANN) are becoming the tool of choice for the pharmaceutical industry due to their ability to mimic the brain's way of functioning. Computational and statistical methods have recently sparked the pharmaceutical industries interest in identifying possible pharmaceutical products that fulfil all technical requirements. Researchers are developing ANN from various scientific areas to overcome prediction, optimization, recognition, and control problems. Conventional techniques can only be used in specific, well-constrained situations. ANN analyses incomplete or unstructured information and converts it into more sensible analysable data by detecting the underlying patterns and similarities. This technology also creates new ideas by rearranging existing knowledge. For example, ANN can construct a promising modelling technique when data sets exhibit nonlinear correlations, which is prominent in pharmaceutical operational processes. In the pharmaceutical industry, this thinking network can be used in disease diagnosis, genomics and proteomics, drug design, to determine physicochemical properties of a drug, drug testing, optimization, pharmacokinetics, in vitro and in vivo correlations, and also to study drug interactions. In this short review article, various applications of
\end{abstract}

ANN in pharmaceutical research are presented.

Key words: Artificial Neural Networks, Pharmaceutical Sciences, Drug Design, Drug Discovery, Drug Delivery, Formulation Development.

\section{${ }^{1}$ Correspondence}

Assoc. Prof. Dr. Mahendran Sekar,

Department of Pharmaceutical Chemistry, Faculty of Pharmacy and Health Sciences, Royal College of Medicine Perak, Universiti Kuala Lumpur, Ipoh-30450, Perak, MALAYSIA.

Email id: mahendransekar@unikl.edu.my

${ }^{2}$ Correspondence

Prof. Dr. TE Gopalakrishna Murthy,

Department of Pharmaceutics, Bapatla College of Pharmacy, Bapatla, Guntur-522101, Andhra Pradesh, INDIA.

Email id: bcp.principal@gmail.com

DOI: 10.5530/jyp.2022.14.2

\section{INTRODUCTION}

The brain is an essential organ in the human body that can control the whole body. It can quickly analyze disordered and unclear information within a short time. It is interconnected with the number of cells, particularly neurons. There are about 1011 neurons in the human brain and about 10000 connections with one another via 1014 synapses-the human brain functions with many yet undiscovered mathematical models involving biological networks. Human brain functionality is the same as one mathematical model: Artificial Neural Networks (ANN) are the type of artificial intelligence system. ANN is the same as the structure of biological neural networks in the human brain. Like, Neurons in the brain are equivalent to a processing element (PE) or artificial node in ANN. ${ }^{1}$

Before knowing more about ANN, it is essential to recognise the worth of artificial intelligence (AI). In the recent decade, researchers have been using AI to drive the emergence of computational precision medicine. AI allows the identification of complex biomarker signatures, i.e. diseasespecific altered networks of genes and proteins shared throughout tumour types and across multi-omics layers, which overcomes the outdated 'one gene, one medication, one illness' paradigm. ${ }^{2}$ Nevertheless, $\mathrm{AI}$ is also being used extensively in precision medicine to construct and optimize diagnosis routes, treatment techniques, and prognoses. This has resulted in a respectable achievement for identifying risk factors for complicated disorders such as cancer by examining gene diversity in an environment. It is also being used to create biomarkers that may be used to stratify patients depending on their sickness risk, prognosis, and/or responsiveness to therapy. The advanced computing capability of using AI for biological data processing is also being used to accelerate the drug development process of precision medicine, ${ }^{3} \mathrm{AI}$ relies on a convergence of technologies with additional. Synergies with life science technology to harness the value of extensive multi-modal data in the form of predictive models supporting decision-making.

Additionally, AI facilitates the design, selection and repurposing of drugs interacting with targets. AI and machine learning (ML) improve medication design and development by understanding disease heterogeneity, discovering dysregulated molecular pathways and therapeutic targets, creating and optimizing drug candidates, and assessing clinical effectiveness in silico. By offering a previously unrivalled degree of understanding of both patient specificities and medication candidate features. ${ }^{4}$

Among the different networks in $\mathrm{AI}$, ANN has a prime role in pharmaceutical drug development applications. ANN is made up of a bunch of interconnected nodes. All of these nodes are useful in

This is an open access article distributed under the terms of the Creative Commons Attribution-NonCommercial-ShareAlike 4.0 License, which allows others to remix, tweak, and build upon the work non-commercially, as long as the author is credited and the new creations are licensed under the identical terms. 
communicating with one another. They are made up of numerous layers called multi-layer perception (MLP). Each layer in this system plays a distinct role in the data it receives. The first of these layers is an input layer, which gets the necessary data. The hidden layers (one or multiple) then collect data from the input layer, process it, and transfer it to the output layer. The hidden layers capture all the minute nuances as they progress deeper. Finally, the output layer produces a result that is straightforward to comprehend. Because it is carried out in parallel, this technique is known as parallel processing. ${ }^{1}$

It is well-known fact that drug discovery and development is a multi-step and expensive process. Since begun of the $20^{\text {th }}$ century, ANN has been used in the field of drug discovery and development effectively. Virtual screening (VS) or high-throughput screening (HTS), formulation development, design of chemical synthesis, docking, quantitative structure-activity relationship (QSAR), ${ }^{5}$ quantitative structure-toxicity relationship (QSTR), pharmacokinetics, and pharmacodynamics, and others have been used with the help of ANN.

ANN can address complicated challenges in the pharmaceutical field by integrating experimental and evidence-based data. They are an effective tool for learning, understanding, and extrapolating data for analysis and prediction. In addition, ANN offers the prediction tool needed to overcome any constraints from the traditional to regression approaches.

\section{TYPES OF NEURAL NETWORKS}

The number of layers between the input and the output of neural networks, also known as hidden layers, which determines the depth of the network. In a feed-forward neural networks (FFNN), information is transmitted in a single path via many input nodes until it reaches the final output node. This network may or may not have hidden node layers to provide more interpretable functionalities. It is used in the processing of huge quantities of data. Recurrent neural networks (RNN) propagate in the same way as FFNN, but they retain all processed input so that it may be utilized in a subsequent phase. Once the network makes an incorrect prediction, the system learns from experience and uses backpropagation to continue working towards the correct prediction. Convolutional neural networks $(\mathrm{CNN})$ is one of the most broadly utilized models nowadays. The computational paradigm of $\mathrm{CNN}$ employs multilayer perceptron and one or more convolutional layers that can be linked or pooled altogether. These convolutional layers generate feature maps, used to record a region of an image broken down into rectangles and sent out for nonlinear processing. Deconvolutional neural networks are used to locate missing features or signals that were previously deemed insignificant to the CNN system's task. Multiple neural networks (MNN) work independently in modular neural networks (no interference with each other's activity throughout the computing process). With this network, complex processes can be completed more quickly. Similarly, with the use of the above-diversified networks, pharmaceutical research is being advanced. A list of ANN uses in pharmaceutical research is provided in Table 1 and Figure 1.

ANN can be employed in drug discovery to drug product development, including analysis and estimation of pharmacokinetic and pharmacodynamic parameters.

\section{ROLE OF ANN IN PHARMACEUTICAL SCIENCES}

\section{ANN in drug discovery}

When it comes to conventional drug development, it is frequently multi-stage, time-consuming, and costly. ANN are helpful to increase the efficiency and speed of new chemical entities (NCEs) design and repurposing of existing drug molecules. Computer-assisted discovery
Table 1: Role of ANN in pharmaceutical sciences.

\begin{tabular}{|c|c|}
\hline $\begin{array}{l}\text { Area in pharmaceutical } \\
\text { sciences }\end{array}$ & Applications of ANN \\
\hline \multirow[t]{5}{*}{ Drug discovery } & $\begin{array}{l}\text { It has been used to assess ligand-receptor } \\
\text { shape complementarity. }\end{array}$ \\
\hline & $\begin{array}{l}\text { Their purpose is to anticipate nonlinear } \\
\text { correlations between physicochemical } \\
\text { characteristics to predict a compound's } \\
\text { biological activity. }\end{array}$ \\
\hline & $\begin{array}{l}\text { Used to estimate the binding energy of the } \\
\text { final docked complex using match surface } \\
\text { characteristics, speeding up the ligand } \\
\text { screening process. }\end{array}$ \\
\hline & $\begin{array}{l}\text { They are used for docking data feature } \\
\text { analysis. }\end{array}$ \\
\hline & $\begin{array}{l}\text { It is used to predict the ADMET properties, } \\
\text { including biotoxicity. }\end{array}$ \\
\hline \multirow{3}{*}{$\begin{array}{l}\text { Pharmaceutical } \\
\text { formulation } \\
\text { development }\end{array}$} & $\begin{array}{l}\text { - They are used in the pre-formulation studies } \\
\text { in formulation development. }\end{array}$ \\
\hline & $\begin{array}{l}\text { It is used to develop formulations containing } \\
\text { multiple active ingredients as well as } \\
\text { controlled-release formulations. }\end{array}$ \\
\hline & $\begin{array}{l}\text { The formulations' alternative to multiple } \\
\text { regression analysis, includes liposomes, } \\
\text { hydrogels, tablets, powders, pellets, } \\
\text { gelispheres, transdermals, granules, and } \\
\text { emulsions. }\end{array}$ \\
\hline \multirow[t]{2}{*}{$\begin{array}{l}\text { In vitro-in vivo } \\
\text { relationship }\end{array}$} & $\begin{array}{l}\text { For example, in vitro qualities and in vivo } \\
\text { patient features have been utilized to predict } \\
\text { clinical outcomes. }\end{array}$ \\
\hline & $\begin{array}{l}\text { It is used to anticipate in vivo outcomes } \\
\text { from in vitro research and to analyze various } \\
\text { product formulations more efficiently. }\end{array}$ \\
\hline $\begin{array}{l}\text { Qualitative and } \\
\text { quantitative drug } \\
\text { analysis }\end{array}$ & $\begin{array}{l}\text { They are used for the identification, } \\
\text { separation, quantification, and optimization } \\
\text { of various analytical conditions. }\end{array}$ \\
\hline \multirow[t]{2}{*}{ PK/PD modelling } & $\begin{array}{l}\text { Useful in PK/PD models with many layers } \\
\text { (input, PK compartment, receptor, and } \\
\text { pharmacological output). }\end{array}$ \\
\hline & $\begin{array}{l}\text { It is used to analyze analytical data, the process } \\
\text { in vitro/in vivo correlations, and generalize } \\
\text { the relationship of drug concentrations with } \\
\text { discovered functional effects. }\end{array}$ \\
\hline Drug-drug interactions & Used to predict potential DDIs. \\
\hline
\end{tabular}

in molecular design, ${ }^{6,7}$ pre-designing of synthetic route, ${ }^{8,9}$ protein structure prediction, ${ }^{10}$ and macromolecular target identification ${ }^{11,12}$ has benefited from deep learning by ANN with multiple processing layers, which has increased the potential and broadened the applicability of computer-assisted discovery. Deep neural networks (DNN) are capable of processing many input characteristics, and the neurons in different layers of a DNN are capable of autonomously extracting information at various hierarchical levels. ${ }^{13}$ Specifically, the DNN model was shown to be the most effective in terms of BEDROC (Boltzmannenhanced discrimination of receiver operating characteristic). Furthermore, it was discovered that multitask and proteochemometric (PCM) implementations outperformed single-task DNN in terms of performance.

DNN models were created by Aliper et al. ${ }^{14}$ to predict pharmacological properties of drugs and possible drug repurposing. Using a variant of the RNN known as UGRNN, Lusci et al. ${ }^{15}$ presented a technique that 


\section{Artificial Neural Networks (ANN)}

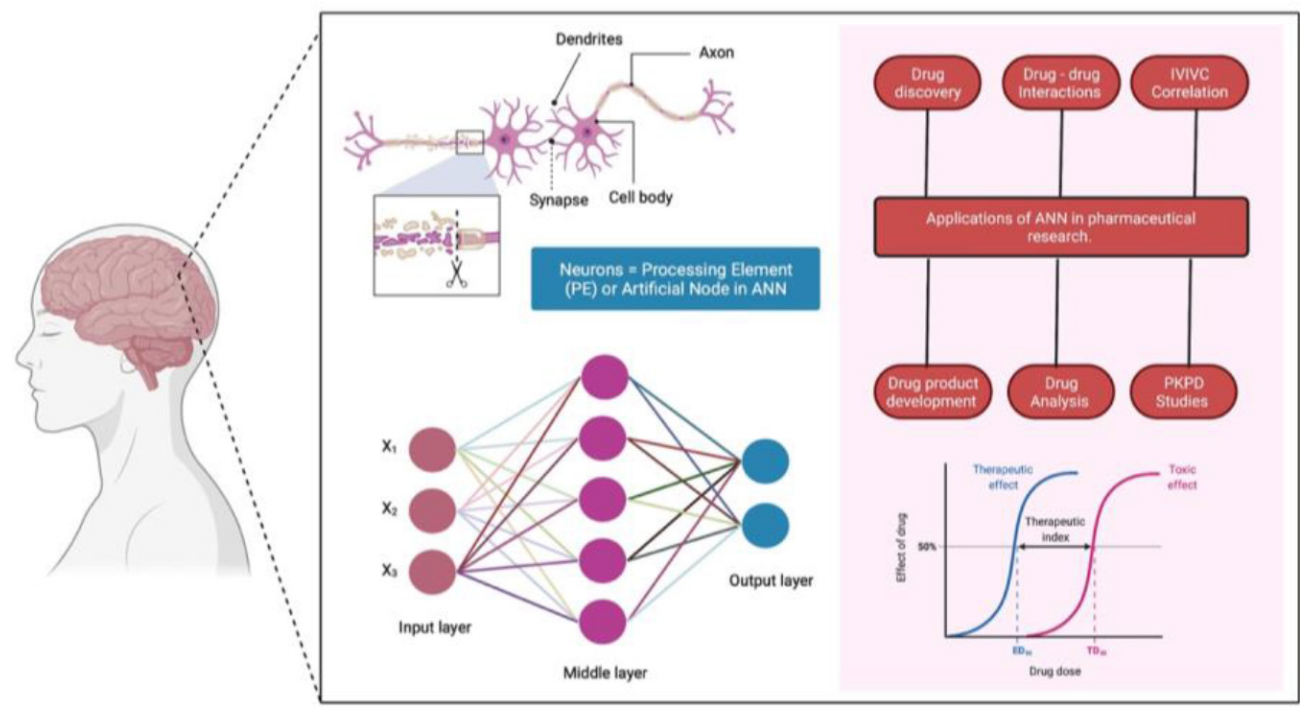

Figure 1: Applications of ANN in pharmaceutical research.

generated predicted solubility models that were similar in accuracy to molecular descriptor-based models while using less computational resources. To anticipate quantum chemical characteristics, Google researchers ${ }^{16}$ rebuilt multiple existing graph convolution methods into a standard framework message passing neural network (MPNN). Bjerrum $^{17}$ used a SMILES string as the input to LSTM RNN to create predictive models without the necessity for molecular descriptions. Gómez-Bombarelli et al. ${ }^{18}$ proposed a novel technique for generating new chemical compounds utilizing a variational autoencoder (VAE).

Olivecrona et al. ${ }^{19}$ used a policy-based reinforcement learning strategy to train RNN and generate molecules with specific user-defined features. Developing NCEs, drawing the chemical structure required to target the receptor, predicting physicochemical properties, enhancing the efficacy of existing drugs, and searching for possible repurposing are all achievable with ANN. ANN also can be used to construct QSAR and quantitative structure-property relationship (QSPR) models that accurately correlate with compounds structure or property descriptor with its chemical or biological activities. Figure 2 summarizes the applications of ANN in drug discovery.

\section{ANN in pharmaceutical formulation development}

The drug substance as such has distinctive features, such as solubility, permeability, stability, processability, and bioavailability, which are not always acceptable in some instances. Pharmaceutical technology enhances a drug substance's inherent qualities by transforming it into an effective pharmaceutical drug product. Pharmaceutical technology requires a strong physicochemical background to describe the pharmaceutical formulations in terms of the properties of their components.

To formulate a dosage form, extensive knowledge is required to decide on the selection of input variables, including its optimisation. The decisions can be made either by using ML or deep learning. ML uses a set of algorithms to look at and understand data. The best decisions are made based on what we've learned from this. Deep learning breaks down the algorithms into many layers in order to make a "artificial neural network." This ANN can learn from the data and make its own intelligent decisions. In contrast to traditional machine learning, deep learning is constantly adapting and improving model efficiency in the face of environmental changes. For example, the solid active pharmaceutical ingredient and other inert additives may exist in amorphous or crystalline forms. Let us think of writing a program that differentiates an amorphous and crystalline form. It looks like a simple task to accomplish, however, it's one that is very hard for a machine to figure out, because we can't just tell a machine what to do by looking at it. A machine can't be able to do this. A few of the amorphous or crystalline solids' properties, such as melting point or solubility, might be mentioned if we were to write a program. What if we used a glassy substance? It's possible that the machine is perplexed! As a result, deep learning is necessary in these situations. It is difficult for a computer to learn in a typical way when additional variables are added, even if the original set of instructions is followed exactly.

In 2004, the Food and Drug Administration (FDA) launched the idea of quality by design $(\mathrm{QbD}){ }^{20}$ It was designed to improve the knowledge of pharmaceutical products and manufacturing processes at all stages of development, including commercial production. The self-learning ANN machines can learn from datasets and model the experimental results based on computer techniques. They employ nonlinear data to analyze interactions between variables, and no assumptions are made about their nature or relevance. ANNs are useful tools for predictive modelling ${ }^{21,22}$ in the development of pharmacological products because they are able to analyze and interpret complicated and hidden patterns in datasets. Neural networks have the benefit of being able to simulate a variety of various response surfaces. As long as there are enough hidden nodes and layers, an ANN can ultimately approximate any surface with precision. However, there is a major drawback: the data are difficult to decipher and need training or experience.

To meet the quality target product profile (QTPP) of the formulation to be developed, critical quality characteristics (CQC) must be identified. The CQC is dependent on critical material attributes (CMA) and critical process parameters (CPP). The trained ANNs can be used as practical pre-formulation tools to generate the fundamental physicochemical properties of the drug. The pharmaceutical product shall satisfy several CQC, and each CQC is dependent on different CMA and CPP. The 


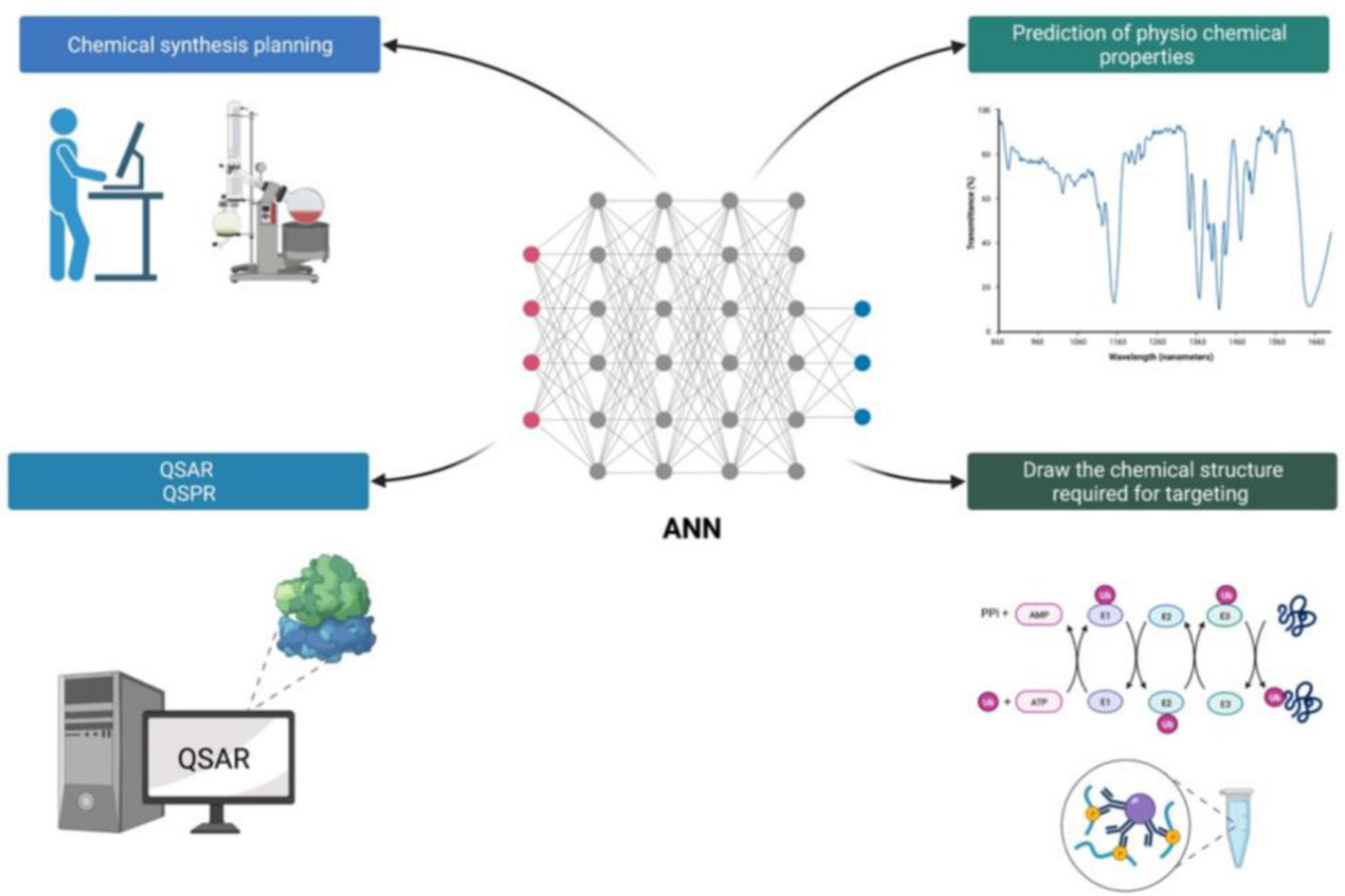

Figure 2: ANN in drug discovery. ANN is practical to draw the structure required for biological activity, prediction of physicchemical properties, synthesis planning, and establishment of QSAR and QSPR.

formulation scientist can develop models to correlate the dependent CQC with the independent CMA and CPP.

The data-driven empirical model simply reflects the current state of knowledge about the problem and serves the most significant role in describing the problem. ANN is used in predictive modelling, and data mining can be used to create an empirical model. Analytical network analysis is used to model relationships of interest in a particular subject by building predictive models to address questions, such as: what would happen to the medication action/quality or stability if the qualitative/ qualitative composition or procedure were changed? Data mining applications include identifying essential variables, the extraction of logical rules (neuro-fuzzy systems), and the creation of response surfaces for a single input variable or a collection of input variables using ANNs. As long as the underlying premise that the significance of inputs can be evaluated by ANN prediction error does not change when a piece of specific information is removed from ANN.

Sensitivity analysis can also be done via the study of derivatives of outputs over the sensitivity of ANN inputs. ${ }^{23,24}$ Regular mathematical models are not suitable to make a decision. For example, a tablet is expected to have a good disintegration with desired hardness. High compression force provides hardness, but it may have low disintegration, and low compression force may produce a soft tablet with good decomposition. The decision to set the compression force is critical, and such decisions may be possible with the predictive models and the sensitivity analysis carried out with ANN. The essential parameters affecting the quality and response can be identified and used for optimization studies.

ANN can be used for the simulation of a process or unit operation. For instance, dry granulation via a roller compactor can be simulated based on the ANN methodology. Two independent process parameters, roll force and screw speed, can be considered as input of the simulation, whereas ribbon density may be considered as output. The deviations may be noticed between the experimental observations and ANN model predicted outputs. Smaller deviations confirm the accuracy of the model. ANNs can be used to develop formulations containing multiple active ingredients as well as controlled-release formulations. The quality target requirements are influenced by critical material and critical process parameters. With its data mining, ANN enables to decide the composition and process to make quality, validated, and effective formulations. The concept of ANN in the development of pharmaceutical products is presented in Figure 3.

In terms of prospects, the combination of liposomes and silicacoated bilayered nanohybrids, referred to as cerasomes, has seen a significant increase in a wide range of scientific implementations drug administration, cancer diagnostics, and treatment. Cerasomes with supramolecular vesicle structures conceptually similar to liposomes can be manufactured using ANN while accounting for all components and factors that went into the final product. When it comes to cancer cell targeting, size matters because the enhanced permeability and retention effect is critical, which takes advantage of cancer cells' higher vascular permeability to allow passive targeting towards the cancer cells.

Thin-film hydration, which disperses liquid in a solution to rehydrate thin film produced after removal of organic solvent, will eventually lead to the formation of spherical vesicles with an aqueous centre (cerosomes). Similarly, in the ultrasonic dispersion method, cerosomes are dispersed in an aqueous medium before being ultrasonicated. Although sonication time and intensity directly influence particle size reduction, other factors such as phospholipid choice and ratio are indirect. In addition, cerasomes can also be surface modified for pegylation or targeting ligands to 


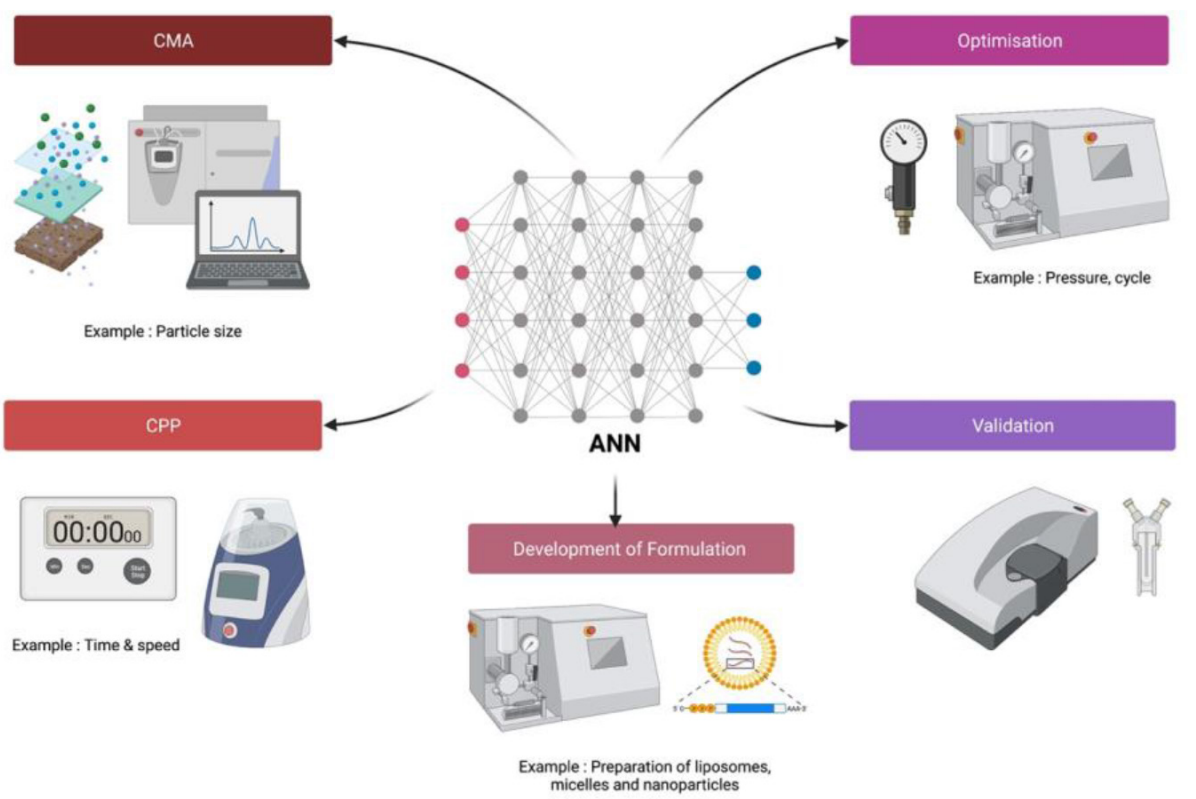

Figure 3: ANN in pharmaceutical formulation development. ANN helps in the formulation development via monitoring validation, optimization, CMA, CPP and others.

\section{Future perspective of drug delivery by using ANN}
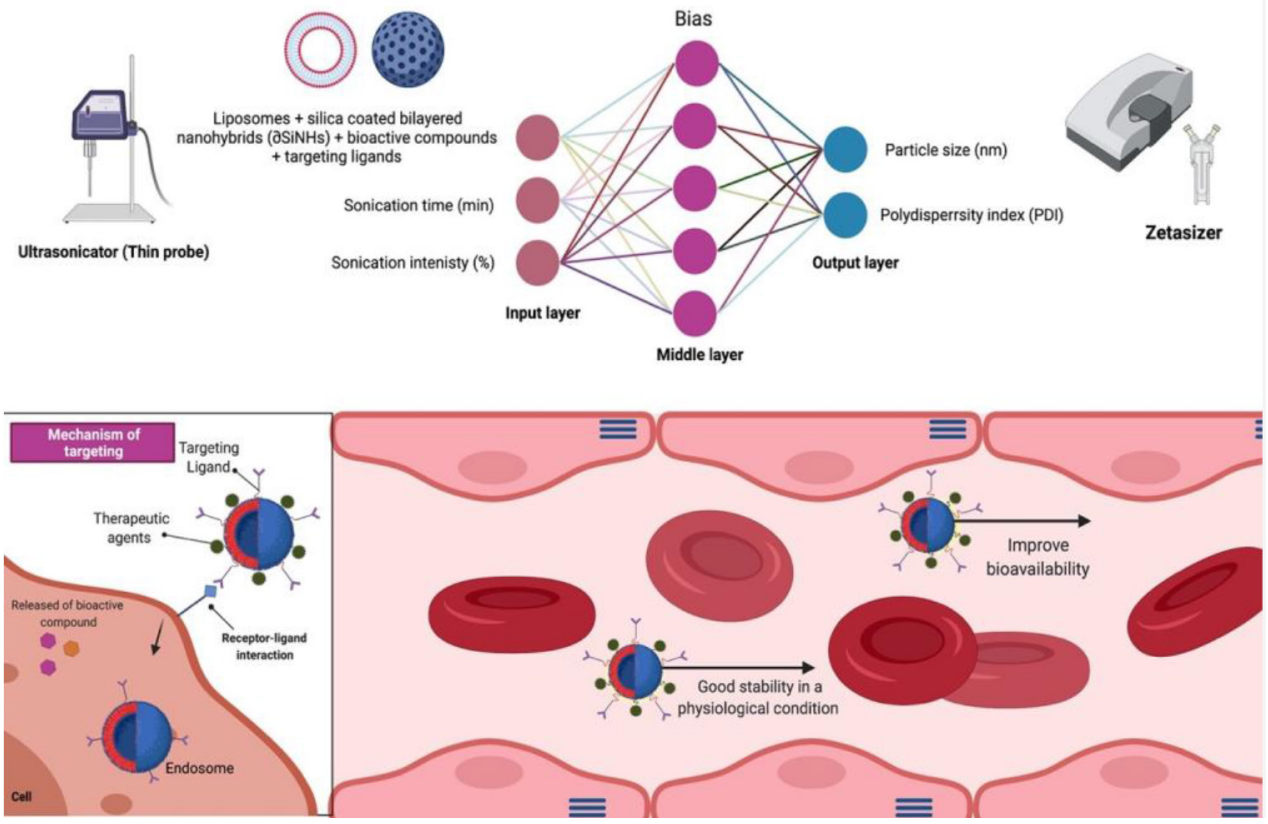

Figure 4: Future perspective of drug delivery by using ANN. The formation of cerosomes is depicted schematically using ANN, where the output can be improved by varying the input components to achieve the desired outcome.

enhance targeting efficacy at the intended site of action. In conclusion, cerasomes may be efficiently designed and developed with optimum sizes by altering component ratios with the help of ANN (Figure 4).

\section{ANN in the development of in vitro-in vivo relationship (IVIVR) model}

IVIVR is used to determine if the relationship between in vitro dissolution and in vivo bioavailability is linear or nonlinear. Without doing animal or human trials, this correlation predicts the bio performance to be examined. Only the input variable linked to dissolution is used in traditional statistical methods. The ANN-based model allows for the inclusion of various factors related to formulation composition and manufacturing process parameters in the model's construction and directly assessing their influence on in vivo responsiveness. It has been shown that these networks may be used as a nonlinear mapping tool for in vitro and in vivo profiles. ${ }^{25-27} \mathrm{~A}$ knowledge database is required 

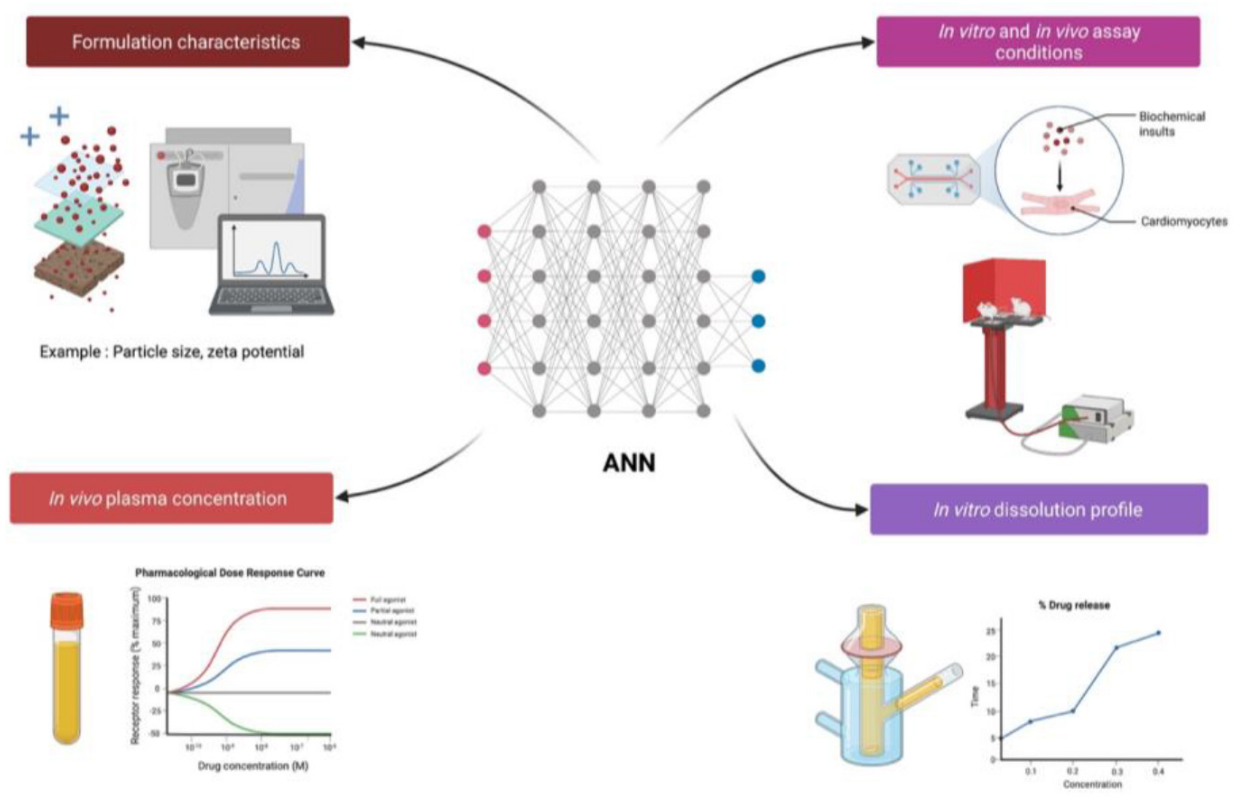

Figure 5: ANN in the development of IVIVC/IVIVR. In vitro-in vivo relationship/correlation is useful for biowaiver and reduces the involvement of time-consuming bioavailability/bioequivalence (BA/BE) studies. Furthermore, ANN predicts the plasma concentration for the changes in physic-chemical, pharmaceutical factors, and in vitro dissolution profile.

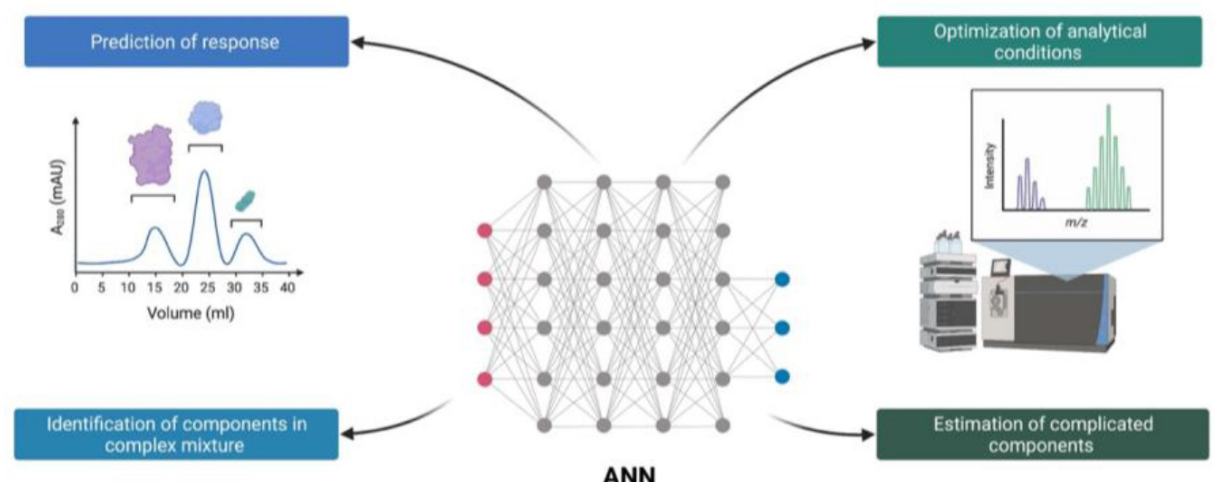

ANN
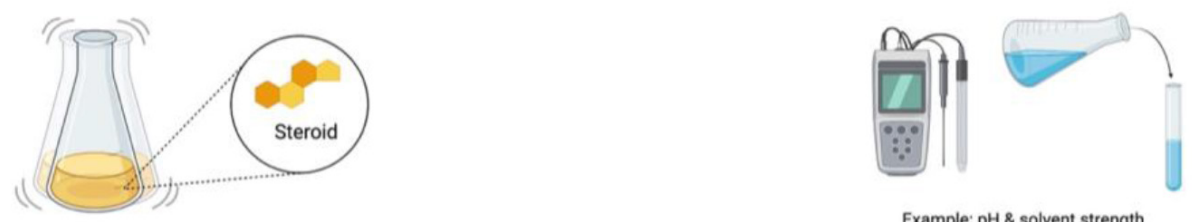

Example: $\mathrm{pH}$ \& solvent strength

Figure 6: ANN in qualitative and quantitative drug analysis. ANN can be used in various qualitative and quantitative drug analyses. It enables the identification, separation, quantification, and optimization of different analytical conditions.

to obtain the data from the published literature or by data mining with computer software. Formulation parameters (type, the qualitative and quantitative content), in vitro dissolution profile, in vivo pharmacokinetic profile, and the conditions under which the test was performed in vitro and in vivo shall be included in the obtained data. Based on dissolution curves, formulation composition, and data on in vivo and in vitro study circumstances, IVIVR neural models can be used to predict total plasma concentration-time profiles. The natural logarithm of plasma concentration is used as the output vector in these models, frequently multiple-input-single-output. As well as the formulation's quantitative and qualitative description, the input vector includes information on the formulation's in vitro dissolution profile, in vivo sampling time, as well as the circumstances of the in vitro and in vivo test.

A backpropagation learning method using multilayer perceptrons is often used. There are a variety of data analysis methods that may be used to examine the data. These include linear activation functions, logistic activation functions, hyperbolic tangent activation functions, and a mixture of logarithmic functions. An ANN architecture can consist of anything from one to several hidden levels. Error measures, such as root mean squared error and normalized base mean squared error 


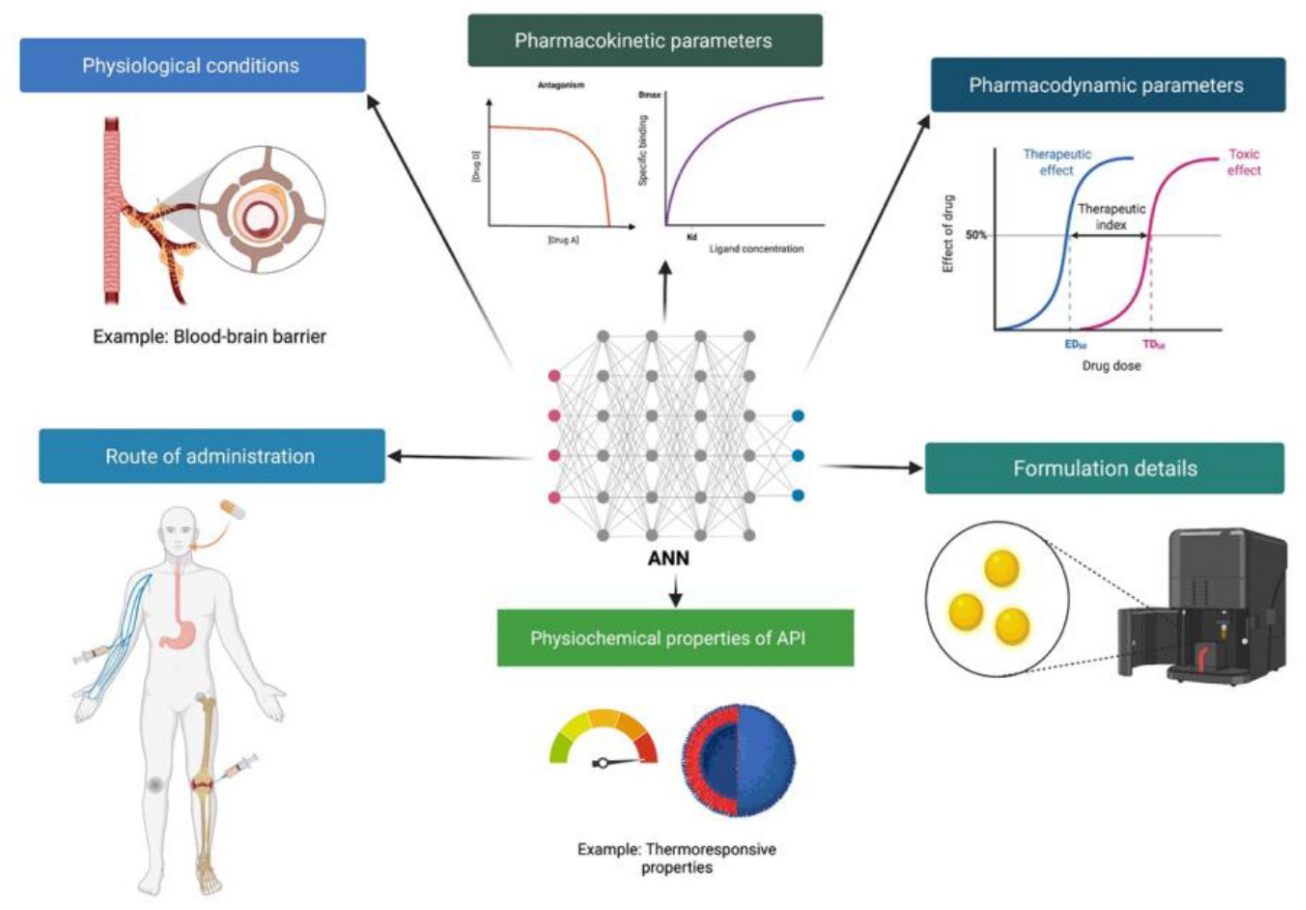

Figure 7: ANN in PK/PD Studies. ANN allows the estimation of PK/PD parameters by simulation of physiological conditions with the given data set.

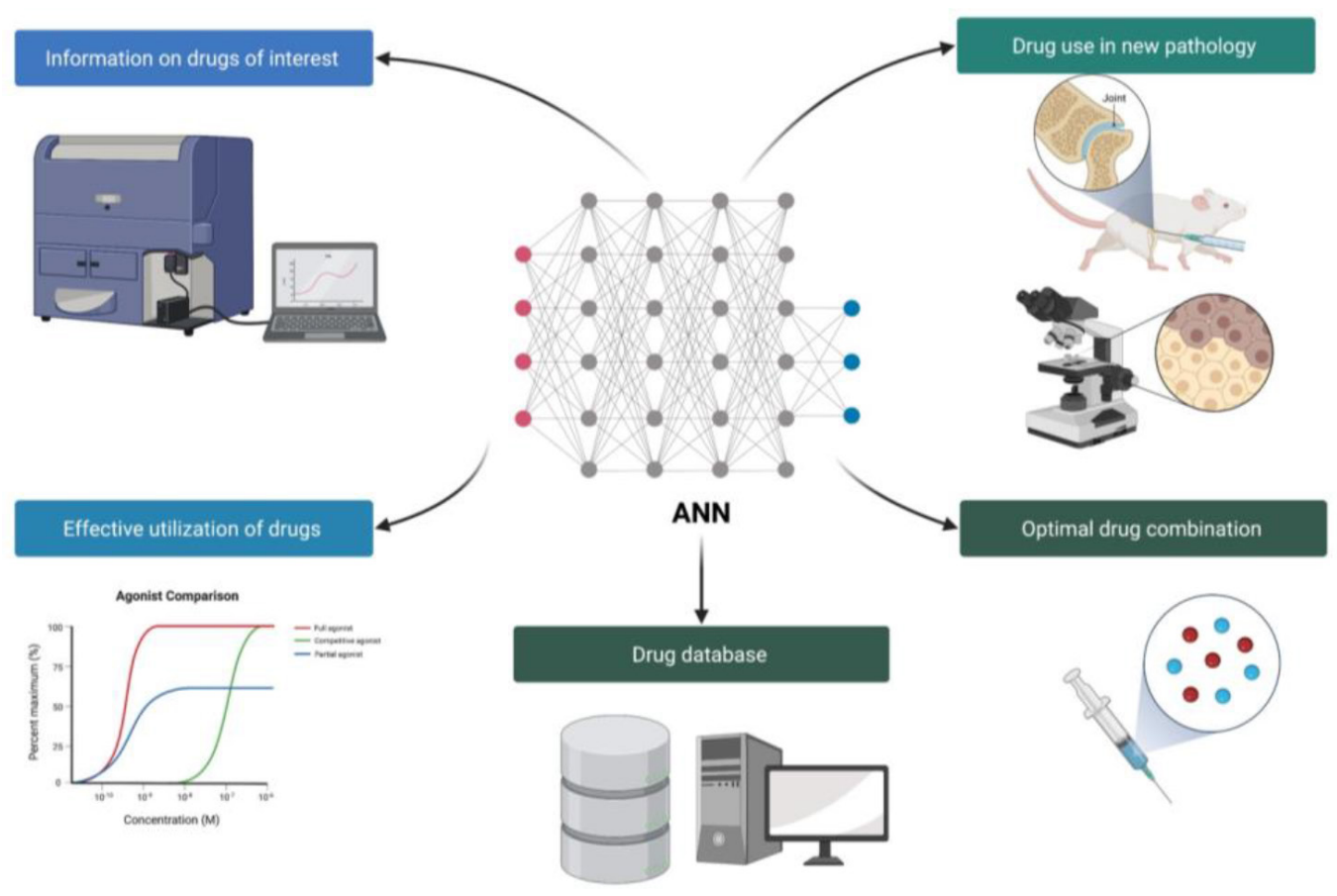

Figure 8: ANN in the prediction of DDI. ANN with its data mining allows the possible prediction of DDI from the drug database.

may be calculated from the expected, observed, observed max, and min data by inserting appropriate mathematical formulae into the data sets. Clinical trials in the pharmaceutical sciences are conducted using the IVIVC/IVIVR method, a standard and essential tool for reducing costs. Its conventional form has only a restricted number of applications. However, the rapid advancement of information technology has resulted in low-cost hardware and superior software, allowing the development of
IVIVR models that are helpful and provide a more significant scientific explanation for decision-making. Figure 5 shows the input and output created by ANN in IVIVC/IVIVR.

\section{ANN in qualitative and quantitative drug analysis}

Impurities that are closely related to the main component and are commonly seen in pharmaceutical dosage forms. Separating and 
quantifying closely related materials is frequently difficult; ANN can discover and estimate such features. Hasani et al. ${ }^{28}$ employed ANN to detect and calibrate tryptophan, tyrosine, and histidine, all of which have comparable structures and spectrums. Due to its ability to recognize nonlinear correlations, ANN is also used to estimate a chiral sample and enantiomeric with a single spectrophotometric measurement. ${ }^{29}$ In chromatography, ANN can be utilized as the foundation for a computer-assisted optimization method to determine optimal gradient settings. ANN can aid in the optimization of highperformance liquid chromatography by creating a connection between solute chromatographic behaviour (capacity factors) and mobile phase composition and $\mathrm{pH}$ to predict retention durations. When the $\mathrm{pH}$ and solvent strength are changed simultaneously, it may use the ANN method to determine component separation. ${ }^{30}$ Thus, ANN can be a helpful tool for reducing the cost and time during the optimization stage of method development. In analyzing complex and complicated data involved in the characterization of mixtures, ANN may aid in detecting relationships. Figure 6 depicts the applications of ANN in drug analysis. Estimating a drug or its metabolite from a biological matrix is a complex process compared to estimating a drug from a formulation and from a dissolution medium as it involves the extraction of the drug from a biological fluid. Therefore, deep learning-based ANN is desired to locate the drug binding site and to select the method to isolate and preserve the drug until analysis. Further, ANN also selects the internal standard and optimises the extraction and estimation.

\section{ANN in pharmacokinetics (PK) and pharmacodynamics (PD) studies}

ANN can be used in a variety of PK/PD studies. It may be used to track various $\mathrm{PK}$ interactions in biological systems and predict characteristics like clearance rates, protein-bound fractions, and volume of distribution. ${ }^{31}$ Drug permeability through biological membranes, such as the stratum corneum and the blood-brain barrier can be predicted using ANN-based models.

Excessive drug metabolism can diminish the drugs effectiveness and cause safety concerns. Several drugs have been removed from the market due to the rise of toxic metabolites in the liver. In addition, drug bioavailability and drug-drug interactions may be affected by metabolic processes. Therefore, pharmacology studies are critical to drug development because they help determine whether and what chemical alterations are required to enhance the metabolic profile of the lead molecule before it can be used as a drug. Rule-based metabolite predictors have been published in the past. ${ }^{32-34}$ With the adoption of deep learning approaches and the availability of enormous chemical reaction datasets, such as the Lowe's dataset, ${ }^{35}$ the accuracy dramatically increased. ${ }^{9,36,37}$ Some of the most widely used drug metabolite prediction tools are SyGMa, GLORYx, BioTransformer, etc.

ANN has been demonstrated to be superior to nonlinear mixedeffects modelling (NONMEM) as an analytical tool for population pharmacokinetic data processing, with lower average absolute errors and average prediction errors. ${ }^{38} \mathrm{~A}$ well-known multilayer feed-forward technique backpropagation ANN (BP-ANN) can be used to create a pharmacokinetic model. Ritschel et $a l,{ }^{39}$ calculated human PK parameters by combining animal PK parameters with physiochemical features (binding of proteins, partitioning coefficients, dissociation constants, total clearance, and distribution volume). In patients with severe diseases, ANN simulation models can be used to predict PK using physiological measurements and can be used to determine the appropriate dose. The development of ANN in drug disposition is a multidisciplinary effort combining biopharmaceutics, PK, pharmacology, and computational biology experts. Traditional pharmacology, including animal investigations, can be reduced using ANN, saving time and money in drug discovery and development. Multi-compartment analysis, physiology, and pathology-based clinical PK use ANN PK models to estimate the optimal dose and dosing frequency based on the patient's condition. Figure 7 depicts the role of ANN in PK/PD studies.

\section{ANN in the prediction of drug-drug interactions (DDI)}

In pharmacology and clinical application, predicting a DDI is a problematic task. Patients and society benefit from the early detection of possible DDI during clinical trials. In silico DDI detection is the most feasible approach to detect interacting drugs. DDI similarities, side-effect similarities, structural similarities, or a mix of similarity metrics are all widely utilized to uncover DDI. Graphical neural networks (GNN) and knowledge graph neural network (KGNN) resolve the DDI prediction. These networks can be used to discover optimal drug combinations, make drugs more effective, and evaluate drug use in new pathologies. The search for drug interactions is essential because multiple drug use can be more harmful than beneficial for many patients. ANN and factor propagation over graph nodes and adjacency matrix factorization (AMF) and adjacency matrix factorization with propagation (AMFP) approaches can be used to forecast DDI. Retrospective analysis can be used to train the nodes using the drug bank database. The DDI extraction assignment has also been reported to be performed using a deep learning strategy of stacked bidirectional long short term memory (Bi-LSTM) and convolutional neural $(\mathrm{CNN})$ networks. ${ }^{40,41}$ The data mining nature of ANN allows for DDI prediction, as shown schematically in Figure 8.

\section{ANN in disease prediction/ diagnosis}

The health care system is now more focused on preventing disease rather than treatment. Therefore, the treatment shall be more accurate, fast and effective. Personalized medicine gains more and more attention as the patients response against different drugs is quite different from person to person. ANN has proven to be a powerful tool to address such issues. ANN can be used to classify a disease (various types of cancer), predict disease risk and its outbreak, as in the case of COVID-19. ANN can be applied to diagnosing breast cancer, heart diseases, ${ }^{42-44}$ kidney diseases,${ }^{45}$ and predicting osteoporosis risk factors and others.

\section{CONCLUSION AND FUTURE PERSPECTIVES OF ANN}

The ANN has several applications in pharmaceutical research. These concepts are applied to drug design and development, including analysis, performance, and interactions if any. The regulatory agencies insist on the manufacturers developing a product with clear scientific evidence and approach, which is necessary for prospects. With the help of ANN, the manufacturer gains time and budget in the development of drug products, and also the patient benefits from an effective, quality, and safe product. ANN fulfils the requirements of the regulatory agency, industry, and the patient.

\section{ACKNOWLEDGEMENT}

The authors acknowledge Universiti Kuala Lumpur Royal College of Medicine Perak, Ipoh, Perak, Malaysia for providing the facilities and services required to complete the study. The Figures in this manuscript have been created with the support of https://biorender.com under a paid subscription.

\section{Definitions}

ANN: Artificial neural networks (ANN), also known as neural networks, are software applications that are modelled after the biological neural networks that constitute animal brains. Artificial neurons are a set of connected units or nodes in an ANN that loosely replicate the neurons in a biological brain.

FFNN: Feedforward neural networks (FFNN) are type of ANN in which nodes do not establish a cycle of connections.

RNN: Recurrent neural networks (RNN) are a type of ANN that is extensively utilised in speech recognition and natural language processing. 
CNN: Convolutional neural networks $(\mathrm{CNN})$ are a type of ANN that is most typically used to evaluate visual information.

MNN: Multiple neural networks (MNN) are a type of ANN that is used to model strongly segregated architectures.

\section{CONFLICT OF INTEREST}

The authors declare that there is no conflict of interest.

\section{REFERENCES}

1. Puri M, Pathak Y, Sutariya VK, Tipparaju S, Moreno W. Artificial neural network for drug design, delivery and disposition. Academic Press; 2015.

2. Azuaje F. Artificial intelligence for precision oncology: Beyond patient stratification. npj Precis Oncol. 2019;3(1):1-5.

3. Saxena A, Chandra S. Artificial intelligence in precision medicine: A perspective in biomarker and drug discovery. Artificial Intelligence and Machine Learning in Healthcare. Springer; 2021;71-88.

4. Moingeon $\mathrm{P}$, Kuenemann M, Guedj M. Artificial Intelligence-enhanced drug design and development: Toward a computational precision medicine. Drug Discov Today. 2022;27(1):215-22. doi: 10.1016/j.drudis.2021.09.006.

5. Mandlik V, Bejugam PR, Singh S. Application of artificial neural networks in modern drug discovery. Artificial Neural Network for Drug Design, Delivery and Disposition. Elsevier; 2016;123-39.

6. Merk D, Friedrich L, Grisoni F, Schneider G. De novo design of bioactive small molecules by artificial intelligence. Mol Inf. 2018;37(1-2). doi: 10.1002/ minf.201700153, PMID 1700153.

7. Zhavoronkov A, Ivanenkov YA, Aliper A, Veselov MS, Aladinskiy VA, Aladinskaya AV, et al. Deep learning enables rapid identification of potent DDR1 kinase inhibitors. Nat Biotechnol. 2019;37(9):1038-40. doi: 10.1038/s41587-019-0224-x.

8. Coley CW, Green WH, Jensen KF. Machine learning in computer-aided synthesis planning. Acc Chem Res. 2018;51(5):1281-9. doi: 10.1021/acs. accounts.8b00087.

9. Schwaller P, Gaudin T, Lányi D, Bekas C, Laino T. 'Found in Translation': Predicting outcomes of complex organic chemistry reactions using neural sequence-tosequence models. Chem Sci. 2018;9(28):6091-8. doi: 10.1039/C8SC02339E.

10. Senior AW, Evans R, Jumper J, Kirkpatrick J, Sifre L, Green T, et al. Improved protein structure prediction using potentials from deep learning. Nature. 2020;577(7792):706-10. doi: 10.1038/s41586-019-1923-7.

11. Jiménez J, Sabbadin D, Cuzzolin A, Martínez-Rosell G, Gora J, Manchester J, et al. PathwayMap: Molecular pathway association with self-normalizing neural networks. J Chem Inf Model. 2019;59(3):1172-81. doi: 10.1021/acs. jcim.8b00711.

12. Öztürk H, Özgür A, Ozkirimli E. DeepDTA: Deep drug-target binding affinity prediction. Bioinformatics. 2018;34(17):i821-9. doi: 10.1093/bioinformatics/bty593.

13. Lee $\mathrm{H}$, Grosse R, Ranganath $\mathrm{R}$, Ng AY. Unsupervised learning of hierarchical representations with convolutional deep belief networks. Commun ACM. 2011;54(10):95-103. doi: 10.1145/2001269.2001295

14. Aliper A, Plis S, Artemov A, Ulloa A, Mamoshina P, Zhavoronkov A. Deep learning applications for predicting pharmacological properties of drugs and drug repurposing using transcriptomic data. Mol Pharmaceutics. 2016;13(7):2524-30. doi: 10.1021/acs.molpharmaceut.6b00248.

15. Lusci A, Pollastri G, Baldi P. Deep architectures and deep learning in chemoinformatics: The prediction of aqueous solubility for drug-like molecules. J Chem Inf Model. 2013;53(7):1563-75. doi: 10.1021/ci400187y.

16. Gilmer J, Schoenholz SS, Riley PF, Vinyals O, Dahl GE, editors. Neural message passing for quantum chemistry. International conference on machine learning; 2017: PMLR.

17. Bjerrum EJ. SMILES enumeration as data augmentation for neural network modeling of molecules. arXiv preprint: 170307076. 2017:1-7.

18. Gómez-Bombarelli R, Wei JN, Duvenaud D, Hernández-Lobato JM, SánchezLengeling B, Sheberla D, et al. Automatic chemical design using a data-driven continuous representation of molecules. ACS Cent Sci. 2018;4(2):268-76. doi: 10.1021/acscentsci.7b00572.

19. Olivecrona M, Blaschke T, Engkvist $\mathrm{O}$, Chen $H$. Molecular de-novo design through deep reinforcement learning. J Cheminform. 2017;9(1):1-14.

20. FDA. Pharmaceutical CGMPs for the $21^{\text {st }}$ Century-a risk-based approach. US Food and Drug Administration; 2004.

21. Achanta AS, Kowalski JG, Rhodes CT. Artificial neural networks: Implications for pharmaceutical sciences. Drug Dev Ind Pharm. 1995;21(1):119-55. doi: 10.3109/03639049509048099

22. LeCun Y, Bengio Y, Hinton G. Deep learning. Nature. 2015;521(7553):436-44. doi: 10.1038/nature14539

23. Behzadi SS, Prakasvudhisarn C, Klocker J, Wolschann P, Viernstein H. Comparison between two types of Artificial Neural Networks used for validation of pharmaceutical processes. Powder Technol. 2009;195(2):150-7. doi: 10.1016/j. powtec.2009.05.025

24. Bourquin J, Schmidli H, Van Hoogevest P, Leuenberger $H$. Comparison of artificial neural networks (ANN) with classical modelling techniques using different experimental designs and data from a galenical study on a solid dosage form. Eur J Pharm Sci. 1998;6(4):287-300. doi: 10.1016/S0928-0987(97)10025-2.

25. De Matas M, Shao Q, Silkstone VL, Chrystyn $\mathrm{H}$. Evaluation of an in vitro in vivo correlation for nebulizer delivery using artificial neural networks. J Pharm Sci. 2007;96(12):3293-303. doi: 10.1002/jps.20965.

26. Dowell JA, Hussain A, Devane J, Young D. Artificial Neural Networks Applied to the in vitro-in vivo Correlation of an Extended-Release Formulation: Initial Trials and Experience. J Pharm Sci. 1999;88(1):154-60. doi: 10.1021/js970148p.

27. Parojcic J, Ibric $S$, Djuric $Z$, Jovanovic $M$, Corrigan OI. An investigation into the usefulness of generalized regression neural network analysis in the development of level A in vitro-in vivo correlation. Eur J Pharm Sci. 2007;30(3-4): 264-72. doi: 10.1016/j.ejps.2006.11.010.

28. Hasani M, Moloudi M, Emami F. Spectrophotometric resolution of ternary mixtures of tryptophan, tyrosine, and histidine with the aid of principal component-artificial neural network models. Anal Biochem. 2007;370(1):68-76. doi: 10.1016/j.ab.2007.06.025.

29. Zhu L, Shabbir SH, Anslyn EV. Two methods for the determination of enantiomeric excess and concentration of a chiral sample with a single spectroscopic measurement. Chem Eur J. 2007;13(1):99-104. doi: 10.1002/ chem.200600402.

30. Lewis JA, Lommen DC, RaddatzWD, Dolan JW, Snyder LR, Molnar I. Computer simulation for the prediction of separation as a function of $\mathrm{pH}$ for reversed-phase high-performance liquid chromatography: I. J Chromatogr A. 1992;592(1-2): 183-95. doi: 10.1016/0021-9673(92)85085-8.

31. Haidar SH, Johnson SB, Fossler MJ, Hussain AS. Modeling the pharmacokinetics and pharmacodynamics of a unique oral hypoglycemic agent using neural networks. Pharm Res. 2002;19(1):87-91. doi: 10.1023/A:1013611617787.

32. Röse P, Gasteiger J. Automated derivation of reaction rules for the EROS 6.0 system for reaction prediction. Anal chim acta. 1990;235:163-8. doi: 10.1016/ S0003-2670(00)82071-1.

33. Salatin TD, Jorgensen WL. Computer-assisted mechanistic evaluation of organic reactions. 1. Overview. J Org Chem. 1980;45(11):2043-51. doi: 10.1021/ jo01299a001.

34. Satoh H, Funatsu K. SOPHIA, a Knowledge Base-Guided Reaction Prediction System - Utilization of a Knowledge Base Derived from a Reaction Database. J Chem Inf Comput Sci. 1995;35(1):34-44. doi: 10.1021/ci00023a005.

35. Lowe DM. Extraction of chemical structures and reactions from the literature. University of Cambridge; 2012.

36. Coley $C$, Jin W, Rogers $L$, Jamison TF, Jaakkola TS, Green WH, et al. A graphconvolutional neural network model for the prediction of chemical reactivity. Chem Sci. 2019;10(2):370-7. doi: 10.1039/C8SC04228D.

37. Schwaller $\mathrm{P}$, Laino T, Gaudin T, Bolgar P, Hunter CA, Bekas C, et al. Molecular transformer: A model for uncertainty-calibrated chemical reaction prediction. ACS Cent Sci. 2019;5(9):1572-83. doi: 10.1021/acscentsci.9b00576.

38. Gobburu JVS, Chen EP. Artificial neural networks as a novel approach to integrated pharmacokinetic_pharmacodynamic analysis. J Pharm Sci. 1996;85(5):505-10. doi: 10.1021/js950433d.

39. Ritschel W, Akileswaran R, Hussain A. Application of neural networks for the prediction of human pharmacokinetic parameters. Methods Find Exp Clin Pharmacol. 1995;17(9):629-43.

40. Lin X, Quan Z, Wang ZJ, Ma T, Zeng X, editors. KGNN: Knowledge graph neural network for drug-drug interaction prediction. IJCAl; 2020.

41. Shtar G, Rokach L, Shapira B. Detecting drug-drug interactions using artificial neural networks and classic graph similarity measures. PLOS ONE. 2019;14(8):e0219796. doi: 10.1371/journal.pone.0219796.

42. Jabbar MA, Deekshatulu BL, Chandra P. Classification of heart disease using artificial neural network and feature subset selection. Glob J Comput Sci Technol. 2013

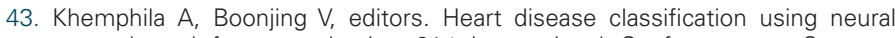
network and feature selection $21^{\text {st }}$ International Conference on Systems Engineering. Vol. 2011. IEEE Publications; 2011.

44. Srinivas K, Rao GR, Govardhan A, editors. Analysis of coronary heart disease and prediction of heart attack in coal mining regions using data mining techniques $5^{\text {th }}$ International Conference on Computer Science and Education. Vol. 2010. IEEE Publications; 2010.

45. Abhishek B, Thakur G, Gupta D. Proposing efficient neural network training model for kidney stone diagnosis. (IJCSIT). Int J Comput. 2012 